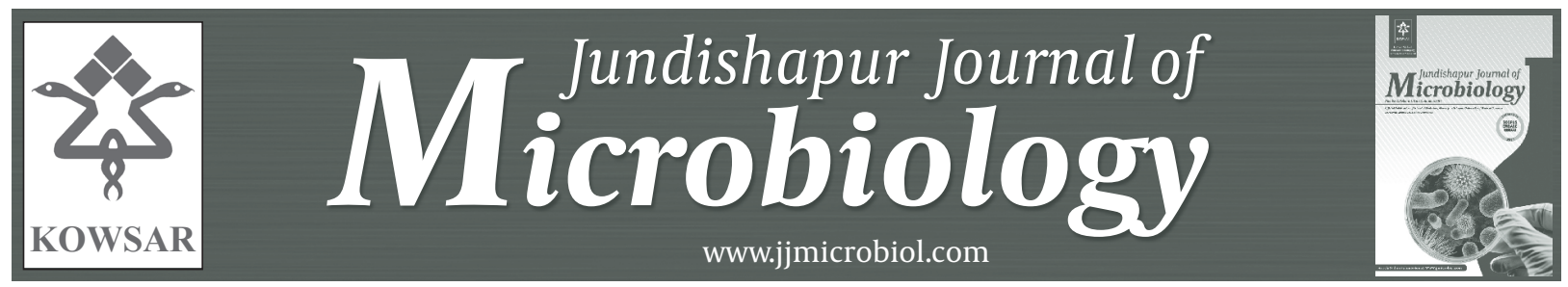

\title{
The Study of Mycobacterium tuberculosis in Iranian Patients With Lung Cancer
}

\author{
Amir Mohamad Hashem Asnaashari ${ }^{1}$, Ali Sadrizadeh ${ }^{2}$, Hamid Ahmadi $^{3}$, Mojtaba Meshkat \\ ${ }^{4}$, Aida Gholoobi ${ }^{5}$, Fariba Rezai Talab ${ }^{6}$, Mahnaz Aamini ${ }^{6}$, Sayed Hosain Ahmadi Hosaini \\ ${ }^{6}$, Davoud Attaran ${ }^{1}$, Mohammad Tohidi ${ }^{1}$, Shahrzad Mohammadzadeh Lari ${ }^{1}$, Reza Basiri ${ }^{1}$, \\ Maryam Sadat Nabavinia ${ }^{5}$, Sina Rostami ${ }^{7}$, Zahra Meshkat ${ }^{8, *}$
}

${ }^{1}$ Department of Pulmonary Diseases, Ghaem hospital, Mashhad University of Medical Sciences, IR Iran

2 Department of Thoracic Surgery, Ghaem hospital, Mashhad University of Medical Sciences, Mashhad, IR Iran

${ }^{3}$ School of Medicine, Mashhad University of Medical Sciences, Mashhad, IR Iran

${ }^{4}$ Isalmic Azad University, Mashhad Branch, Mashhad, IR Iran

${ }^{5}$ Microbiology and Virology Research Center, Mashhad University of Medical Sciences, Mashhad, IR Iran

${ }^{6}$ Department of Pulmonary Diseases, Imam Reza hospital, Mashhad University of Medical Sciences, IR Iran

${ }^{7}$ School of Sciences, Ferdowsi University of Mashhad, Mashhad, IR Iran

${ }^{8}$ Women's Health Research Centre, Mashhad University of Medical Sciences, Mashhad, IR Iran

*Corresponding author Zahra Meshkat, Women's Health Research Centre, Mashhad University of Medical Sciences, Mashhad, Iran. Tel: +98-5118012453; Fax: +98-5118002960, E-mail: meshkatz@mums.ac.ir.

\section{A B S T R A C T}

Background: Mycobacterium tuberculosis has the ability to invade type II alveolar epithelial cells. As a result, the associations between invasion of alveolar epithelial cells and pathogenesis of lung infection seem strong.

objectives: The current study aimed to evaluate the presence of $M$. tuberculosis in patients with lung cancer.

Patients and Methods: This cross-sectional study was performed on samples collected from 380 patients with lung cancer who referred to two state-run hospitals in Mashhad, Iran. Microscopic and cultural methods were utilized to assess the presence of M. tuberculosis in the patients`specimens.

Results: The subjects included 252 (66.3\%) males and 128 (33.7\%) females. Based on cultural and microscopic methods, M. tuberculosis infection was observed in twenty six $(6.8 \%)$ of cases.

Conclusions: Results of the current study showed the high prevalence of M. tuberculosis among the patients with lung cancer; therefore, it seems that continuous surveillance is essential to monitor the M. tuberculosis in the patients with lung cancer.

Keywords: Mycobacterium tuberculosis; Lung Cancer; Epidemiology

Copyright (C) 2013, Ahvaz Jundishapur University of Medical Sciences; Published by Kowsar Corp.

-Article type: Research Article; Received: 27 Mar 2012, Revised: 19 Jun 2012, Accepted: 03 Jul 2012; DOI: 10.5812/jjm.4993

-Implication for health policy/practice/research/medical education:

It seems necessary for clinicians and health care systems to be fully aware of Mycobacterium tuberculosis in patients with lung cancer and continuous surveillance is essential to monitor the patients for proper management.

PPlease cite this paper as:

Hashem Asnaashari AM, Sadrizadeh A, Ahmadi H, Meshkat M, Gholoobi A, Rezai Talab F, et al. The Study of Mycobacterium tuberculosis in Iranian Patients With Lung Cancer. Jundishapur J Microbiol. 2013;6(3):237-41. DOI: 10.5812/jjm.4993

Copyright (C) 2013, Ahvaz Jundishapur University of Medical Sciences; Published by Kowsar Corp.

This is an Open Access article distributed under the terms of the Creative Commons Attribution License (http://creativecommons.org/licenses/by/3.0), which permits unrestricted use, distribution, and reproduction in any medium, provided the original work is properly cited. 


\section{Background}

Worldwide, lung cancer constituted 13\% (1.6 million) of the total cases of cancer in 2008(1). Lung and bronchus cancers caused death of an estimated 156,940 in the United States in 2011(2). In Iran, it is the fifth most common cause of cancer and according to the two reports published in 2002 and 2003, its prevalence was considered to be very low $(3,4)$.

Overall, it is estimated that one-third of the world's population is infected with Mycobacterium tuberculosis (MTB). The incidence is directed toward Asia, where China, India, Bangladesh, Indonesia and Pakistan constitute over $50 \%$ of the total cases (5). In Iran, in 2005 it was reported that the prevalence of tuberculosis (TB) was high in border-line provinces such as Sistan and Balouchestan, Golestan, Khorasan, Hormozgan and Khouzestan (6).

Several studies have explained the possible mechanisms behind the role of MTB infection and lung cancer in inducing genetic damage. Nalbandian et al. findings in 2009 provided evidence for the role of chronic MTB infection in cell dysplasia and squamous cell carcinoma (SCC) (7). Lung cancer is supposed to be related to pulmonary inflammation; since inflammatory cells release activated oxygen molecules, inflammatory mediators and proteolytic enzymes promote molecular changes leading to cancer (8). It was suggested that TB intensifies the probability of lung cancer due to the fact that inflammation and fibrosis may result in genetic damage (9).

\section{Objectives}

The current study was conducted to assess the presence of M. tuberculosis in patients with lung cancer.

\section{Patients and Methods}

This cross-sectional study was carried out on samples collected from 380 patients with lung cancer who referred to two state-run hospitals in Mashhad between May 2009 and October 2011. Patients underwent bronchoscopy and evidence for lung cancer was observed. Informed consent was obtained from participants. After preparing bronchoalveolar lavage fluid for pathological observations to confirm the previous diagnosis, extra lavage fluid was employed in the study for microscopic investigation and also for the bacterial culture. It is critical to note that no extra samples were obtained for the research. One hundred and thirty seven of the total cases lack cytological results. Regarding the fact that verifying lung cancer requires cytological studies by at least two pathologists, two surveys were employed. Initially, 243 patients with thorough cytological results, and subsequently, the total 380 patients were examined.

For bacteriological tests, samples were decontaminated and homogenized with equal amount of $4 \% \mathrm{NaOH}$. After neutralizing procedures by $2 \mathrm{~N} \mathrm{HCl}$, five drops of re-suspended sediments were subsequently inoculated to Lowenstein-Jensen solid medium and then incubated in a slanted position for about two months. Two drops of the sediments were used for indirect smear preparation (Ziehl- Neelsen staining). Slide reading followed the recommendations outlined in World Health Organization (WHO) guidelines and Mycobacteria confirmed with phenotypic results.

Statistical analysis was performed using Pearson's Chi-square and in cases when more than $20 \%$ of the expected frequencies were less than five, Fisher's exact test was used. In order to interpret multivariate statistics, Logistic regression was carried out. SPSS v.20 (SPSS Inc., Chicago, IL, USA) was employed for the statistical analysis. Differences with $P<0.05$ were considered to be statistically significant.

\section{Results}

In the current study, 380 patients clinically diagnosed with lung cancer were employed. The subjects included 128 (33.7\%) female and 252 (66.3\%) male. M. tuberculosis was positive by culture in 26 cases out of 380 .

No significant statistical differences were observed between the culture results and sex ( $P$ value $=0.068)$, age groups $(P$ value $=0.637)$, Marital status $(p$-value $=0.170)$, Literacy $(P$ value $=0.223)$, Smoking $(P$ value $=0.476)$ (Table 1). The data showed statistically significant differences between addiction and the positive results in culture method (P value $=0.007$ ) (Table 1 ). Nineteen (5\%) of the total subjects were taking anti-tuberculosis drug, whereas 361 (95\%) were non-users and the differences were not statistically significant $(P$ value $=0.999)$. In the current study, the correlation between culture and microscopic methods indicated $57.8 \%$ consistency which was statistically significant $(P$ value $=0.0001)($ Table 2 ). The sensitivity, specificity, agreement and disagreement of the microscopic method in comparison with culture method were $69.2 \%, 98.5 \%, 54.5 \%$ and $97.7 \%$, respectively.

\section{Discussion}

Although some studies such as those reported in Canada in 1986 and 2001 suggested no significant relationship between $M$. tuberculosis and lung cancer, and also lung and heart diseases (10), several studies pointed out the existence of association between M. tuberculosis and lung cancer. According to a study by Kurasawa in 1992, lung cancer is 1-2\% linked with tuberculosis (11). However, a similar study conducted by Tamura, showed a linkage of 1-5\% (12). 
Table 1. Socio-Demographic Data of the Patients With Lung Cancer Based on Positive/Negative Results of the Bacteria Culture

\begin{tabular}{|c|c|c|c|c|}
\hline Socio-demographic variables & Number & Number of positive culture, No. (\%) & Number of negative culture, No. (\%) & $P$ value \\
\hline \multicolumn{5}{|l|}{ Sex } \\
\hline Male & $252(66.3)$ & $13(5.2)$ & $239(94.8)$ & 0.068 \\
\hline Female & $128(33.7)$ & $13(10.2)$ & $115(89.8)$ & 0.068 \\
\hline Total & $380(100)$ & $26(6.8)$ & $354(93.2)$ & 0.068 \\
\hline \multicolumn{5}{|l|}{ Age, $y$} \\
\hline$<30$ & $10(2.63)$ & $0(0.0)$ & $10(100.0)$ & 0.637 \\
\hline $30-39$ & $15(3.95)$ & $0(0.0)$ & $15(100.0)$ & 0.637 \\
\hline $40-49$ & $38(10.00)$ & $1(2.6)$ & $37(97.4)$ & 0.637 \\
\hline $50-59$ & $95(25.00)$ & $6(6.3)$ & $89(93.7)$ & 0.637 \\
\hline$>59$ & $222(58.42)$ & $19(8.6)$ & $203(91.4)$ & 0.637 \\
\hline Total & $380(100)$ & $26(6.8)$ & $354(93.2)$ & 0.637 \\
\hline \multicolumn{5}{|l|}{ Marital status } \\
\hline Single & $8(2.1)$ & $1(12.5)$ & $7(87.5)$ & 0.170 \\
\hline Married & $352(92.6)$ & $22(6.3)$ & $330(93.8)$ & 0.170 \\
\hline Divorced/widowed & $20(5.3)$ & $3(15.0)$ & $17(85.0)$ & 0.170 \\
\hline Total & $380(100)$ & $26(6.8)$ & $354(93.2)$ & 0.170 \\
\hline \multicolumn{5}{|l|}{ Literacy } \\
\hline Illiterate & $204(53.7)$ & $14(6.9)$ & $190(93.1)$ & 0.223 \\
\hline Elementary school & $111(29.2)$ & $11(9.9)$ & $100(90.1)$ & 0.223 \\
\hline Secondary school & $24(6.3)$ & $0(0.0)$ & $24(100.0)$ & 0.223 \\
\hline High school diploma & $29(7.6)$ & $0(0.0)$ & $29(100.0)$ & 0.223 \\
\hline Academic education & $12(3.2)$ & $1(8.3)$ & $11(91.7)$ & 0.223 \\
\hline Total & $380(100)$ & $26(6.8)$ & $354(93.2)$ & 0.223 \\
\hline \multicolumn{5}{|l|}{ Drug addiction } \\
\hline Drug addicts & $120(31.6)$ & $2(1.7)$ & $118(98.3)$ & $0.007^{\mathrm{a}}$ \\
\hline Non-drug addicts & $260(68.4)$ & $24(9.2)$ & $236(90.8)$ & $0.007^{\mathrm{a}}$ \\
\hline Total & $380(100)$ & $26(6.8)$ & $354(93.2)$ & $0.007^{\mathrm{a}}$ \\
\hline \multicolumn{5}{|l|}{ Smoking } \\
\hline Smokers & $201(52.9)$ & $12(6.0)$ & $189(94.0)$ & 0.476 \\
\hline Non-smokers & $179(47.1)$ & $14(7.8)$ & $165(92.2)$ & 0.476 \\
\hline Total & $380(100)$ & $26(6.8)$ & $354(93.2)$ & 0.476 \\
\hline
\end{tabular}

a Statistically significant

Table 2. Consistency Results For Direct Microscopic Method in Comparison With the Culture Method in Patients With Clinical Lung Cancer Manifestations

\begin{tabular}{llll}
\hline Results $^{\text {a, b }}$ & Positive, No. (\%) & Negative, No. (\%) & Total, No. (\%) \\
\hline Positive & $18(69.2)$ & $15(4.2)$ & $33(8.7)$ \\
Negative & $8(30.8)$ & $339(95.8)$ & $347(91.3)$ \\
Total & $26(100)$ & $354(100)$ & $380(100)$ \\
\hline
\end{tabular}

a Probability $=$ Measurement of Agreement (Kappa) $=0.578$

$\mathrm{b}_{\mathrm{P} \text { value }}=0.0001$

A study carried out by $\mathrm{Wu}$, suggested that infection with M. tuberculosis increases the risk of developing lung 
cancer (13). Another study by Yu et al. concluded the same notes and indicated that the incidence of lung cancer in patients with $M$. tuberculosis is 10.9 times more than that of non-tuberculosis subjects (14).

In the present study, positive $M$. tuberculosis culture was found in $6.8 \%$ of total samples, which was more than that of Kurasawa (1-2\%) (11), Tamura (1-5\%) (12) and Cicenas (2.1\%) (15). This was probably caused by samples taken from low socioeconomic families because they live in areas with lower sanitation and have populated family environment with limited house space.

Engels et al. pointed out that the increase in risk of developing lung cancer following infection with M.tuberculosis is biologically conceivable. Since respiratory symptoms are present months before clinical manifestations, during which, M. tuberculosis induces production of tumor necrosis factors (TNFs) by leukocytes. Immune responses, therefore, generate various types of oxygen and nitrogen molecules that in turn may bind to DNA and alter the genome (16).

Cicenas et al. believe that the reason behind coexistence of tuberculosis and lung cancer in patients should be divided into three categories: 1 . No association exists between tuberculosis and lung cancer. 2. Alterations made by tuberculosis lead to deformation of alveoli and bronchiole as well as dysplasia of respiratory epithelial cells. This, in turn, would raise the probability of developing lung cancer. 3. During the process of lung cancer progression, an old foci of tuberculosis reactivates and leads to dissemination of M. tuberculosis (15).

In the present study, $83.4 \%$ of the subjects were in the age group 50-59 and over 60 . This is justifiable considering the high incidence of lung cancer between the age 55 and 65 and the current study results is similar to those of previous studies; the study conducted in 2011 by Wang et al. put forward the notion that regarding each region, the age group accounting for much of the burden of lung cancer differs and that the lung cancer was most prevalent in age groups 50-59, 60-69 and 70-79 years old (17). Similar results were observed in a study by Schottenfeld which indicated that most cases of lung cancer were patients aged 60-80 (18).

Totally, $82.7 \%$ of our subjects had low education level. This fact could be interpreted in two ways; either the subjects referred to state-run hospitals, where the current study was conducted, because of their low socioeconomic status, or that the risk of lung cancer among low socioeconomic class has elevated. Dalstra et al. reported that cancer, in general, had a higher frequency among lower educated subjects when the age group 25-59 was taken into account which is similar to the results of the current study; however, the trend was opposite among those of 60-79 age group (19). According to the study by Devesa and Diamond, no strong association was found between education and elevated risk of lung cancer among fe- males of both races (black and white) (20).

The current study is the first study in Iran which considers M. tuberculosis frequency in patients with lung cancer and can be a turning point for such studies. In comparison with other cross-sectional studies carried out in the world, the sample size recruited in the current study was acceptable. The researchers suggest bronchoalveolar lavage specimen as an appropriate alternative to sputum specimen because in the former, no extra sample is obtained from the patients.

This study had crucial limitations such as being crosssectional, therefore not proposing a cause and effect relationship between lung cancer and M. tuberculosis, and also there was insufficient access to histology documents of patients. Another limitation was that samples were taken solely from two state-run hospitals and were not random. The present study encourages further investigations, such as cohort and case control studies, to reveal stronger evidence supporting the associations between these two significant diseases.

\section{Acknowledgements}

This study was a thesis presented for the degree of Medical Doctor (MD) in Mashhad University of Medical Sciences (Thesis No. 6587).

\section{Financial Disclosure}

There is no Financial Disclosure.

\section{Funding/Support}

This study was supported by Mashhad University of Medical Sciences, Mashhad, Iran (grant No. 87571).

\section{Authors' Contribution}

None declared.

\section{References}

1. Jemal Ahmedin, Bray Freddie, Center Melissa M, Ferlay Jacques, Ward Elizabeth, Forman David. Global cancer statistics. CA: A Cancer J clinicians. 2011;61(2):69-90

2. Siegel Rebecca, Ward Elizabeth, Brawley Otis, Jemal Ahmedin. Cancer statistics, 2011. CA: a cancer journal for clinicians. 2011;61(4):212-236

3. Hosseini M, Alinaghi SAS, Naghan PA, Karimi S, Bahadori M, Khodadad $\mathrm{K}$, et al. A clinicopathologic study of lung cancer cases in Iran. Tanaffos. 2009;8(3):28-36

4. Sadjadi A, Nouraie M, Mohagheghi MA, Mousavi-Jarrahi A, Malekezadeh R, Parkin DM. Cancer occurrence in Iran in 2002, an international perspective. Asian Pac J Cancer Prev. 2005;6(3):359-63

5. Dye C, Scheele S, Dolin P, Pathania V, Raviglione MC. Consensus statement. Global burden of tuberculosis: estimated incidence, prevalence, and mortality by country. WHO Global Surveillance and Monitoring Project. JAMA. 1999;282(7):677-86

6. Salek S, Emami H, Masjedi MR, Velayati AA. Epidemiologic status of tuberculosis in Golestan province. Tanaffos. 2008;7(3):63-68

7. Nalbandian A, Yan BS, Pichugin A, Bronson RT, Kramnik I Lung carcinogenesis induced by chronic tuberculosis infection: the experimental model and genetic control. Oncogene. 
2009;28(17):1928-1938

8. Smith CJ, Perfetti TA, King JA. Perspectives on pulmonary inflammation and lung cancer risk in cigarette smokers. Inhal Toxicol. 2006;18(9):667-677

9. Ballaz S, Mulshine JL. The potential contributions of chronic inflammation to lung carcinogenesis. Clin Lung Cancer. 2003;5(1):46-62

10. Ramanakumar AV, Parent ME, Menzies D, Siemiatycki J. Risk of lung cancer following nonmalignant respiratory conditions: evidence from two case-control studies in Montreal, Canada. Lung Cancer. 2006;53(1):5-12

11. Kurasawa T, Takahashi M, Kuze F, Amitani R, Murayama T, Suzuki $\mathrm{K}$, et al. A clinical study on coexistence of active pulmonary tuberculosis and lung cancer]. Kekkaku:[Tuberculosis].1992;67(2):119

12. Tamura A, Hebisawa A, Tanaka G, Tatsuta H, Tsuboi T, Nagai H, et al. Active pulmonary tuberculosis in patients with lung cancer]. Kekkaku:[Tuberculosis].1999;74(11):797

13. Wu CY, Hu HY, Pu CY, Huang N, Shen HC, Li CP, et al. Pulmonary tuberculosis increases the risk of lung cancer. Cancer. 2011;117(3):618-24

14. Yu YH, Liao CC, Hsu WH, Chen HJ, Liao WC, Muo CH, et al. In- creased Lung Cancer Risk among Patients with Pulmonary Tuberculosis: A Population Cohort Study. J Thorac Oncol. 2011;6(1):32

15. Cicènas S, Vencevičius V. Lung cancer in patients with tuberculosis. WJ Surg Oncol. 2007;5(1):22

16. Engels EA, Shen M, Chapman RS, Pfeiffer RM, Yu YY, He X, et al Tuberculosis and subsequent risk of lung cancer in Xuanwei, China. Int J Cancer. 2009;124(5):1183-1187

17. Wang C, Li X, Dai L, Zhang G, Gao L, Zhao Q, editors.Analysis of lung cancer gender differences and age structure in the high prevalence areas of Xiamen. 2011; IEEE;

18. Schottenfeld D. Epidemiology of lung cancer. Lung cancer: principles and practice.1996:305-321

19. Dalstra JAA, Kunst AE, Borrell C, Breeze E, Cambois E, Costa G et al. Socioeconomic differences in the prevalence of common chronic diseases: an overview of eight European countries. Int $J$ Epidemiol. 2005;34(2):316

20. Devesa SS, Diamond EL. Socioeconomic and racial differences in lung cancer incidence. American J Epidemiol.1983;118(6):818 